\title{
Remifentanil attenuates the spindle activity and decrease EEG amplitude during propofol anesthesia
}

\author{
Hagihira $\mathrm{S}^{1,2}$, Kang $\mathrm{H}^{2}$, Takashina $\mathrm{M}^{3}$, Mori $\mathrm{T}^{4}$
}

\section{Dept of Anesthesiology, Kansai Medical University, Hirakata City, Japan. ${ }^{2}$ Dept of Anesthesiology \& Intensive Care Medicine, Osaka University Graduate School of Medicine, Suita City, Japan. ${ }^{3}$ Osaka University Hospital, Surgical Center, Suita City, Japan. ${ }^{4}$ Dept of Medical Informatics, Osaka General Medical Center, Osaka, Japan.}

Background: It is generally thought that opioids have little effect on EEG, especially when noxious stimuli were not added. Table 1 showed the changes of EEG amplitude, SEF95, BIS 5 minutes after bolus administration of fentanyl $3 \mu \mathrm{g} / \mathrm{kg}$ during sevoflurane anesthesia before incision. None of them changed after fentanyl administration. However, we felt that EEG amplitude (Amp) seemed to become smaller after starting the infusion of remifentanil during propofol anesthesia. To examine this, we compared the Amp observed without remifentanil and that with remifentanil during propofol anesthesia.
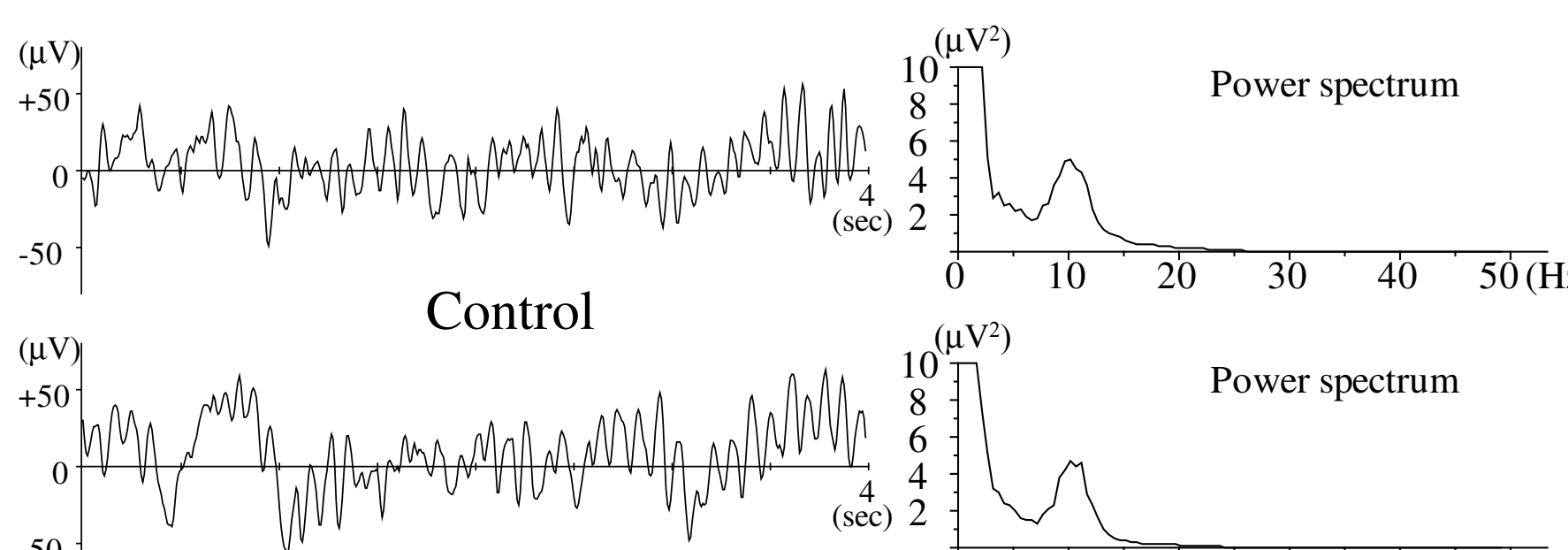

After fentanyl

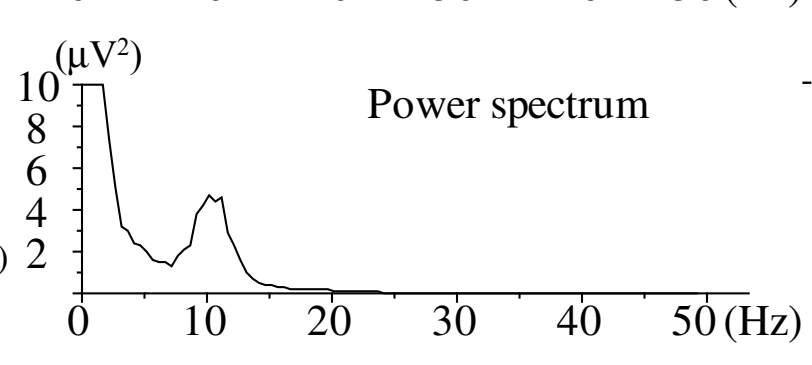

[Fig 1] Effects of fentanyl on EEG during sevoflurane anesthesia in $34 \mathrm{yr}$ old female

\begin{tabular}{|l|c|c|c|}
\hline & Amplitude $(\mu \mathrm{V})$ & SEF95 $(\mathrm{Hz})$ & BIS \\
\hline Control & $15.8 \pm 4.2$ & $13.4 \pm 1.2$ & $42.1 \pm 4.8$ \\
\hline After fentanyl & $16.0 \pm 4.1$ & $13.3 \pm 1.2$ & $42.5 \pm 5.7$
\end{tabular}

[Table 1] Effects of fentanyl on EEG during sevoflurane anesthesia $(N=12)$ Anesthesiology 2004;100:818-25

Materials and Methods: We analyzed the EEG data obtained in the previous study. After approval of the ethical committee of our institute and obtained written informed consent from the participants, we enrolled 26 female patients (aged 33-65) who were scheduled mammectomy for breast cancer. Besides the standard monitors, we used BIS monitor (BIS-XP) and all raw EEG packet as well as EEG derived parameters were recorded on a computer using our original software "BSA for BIS". We defined the amplitude of EEG as the half of the voltage difference between the adjacent two local peaks, and Amp was defined as the average of EEG amplitude in the latest 20 seconds of EEG signals. Propofol was infused using target-controlled infusion (TCI) pump (TE-371; TERUMO, TOKYO, JAPAN). We determined Amp without remifentanil and that with remifentanil infused at $0.2 \mu \mathrm{g} / \mathrm{kg} / \mathrm{min}$ at the same effect-site concentration of propofol before incision. We also compared the power spectrum of EEG, with or without remifentanil. The propofol concentration was adjusted to surgical level of anesthesia in each patient by EEG monitoring.

Data were analyzed by paired t-test. P values of less than 0.05 were considered significant.

\begin{tabular}{|l|r|} 
& Mean \pm SD $($ range $)$ \\
\hline Age $(\mathbf{y r})$ & $52.8 \pm 9.0(33-65)$ \\
\hline Weight $(\mathrm{kg})$ & $55.3 \pm 7.9(43.0-70.0)$ \\
\hline Height $(\mathrm{cm})$ & $158.8 \pm 4.1(\mathbf{1 5 1 . 5 - 1 6 7 . 0})$ \\
\hline
\end{tabular}

Results: Table 2 showed the demographic data. Fig 2 showed the EEG waveforms and power spectrums before and after remifentanil infusion. As shown in this figure, Amp became smaller and $\alpha$ power $(8-13 \mathrm{~Hz})$ also became smaller after remifentanil infusion. And both Amp and $\alpha$ power significantly decreased after remifentanil infusion $(p<0.001)$ as shown in Table 3.
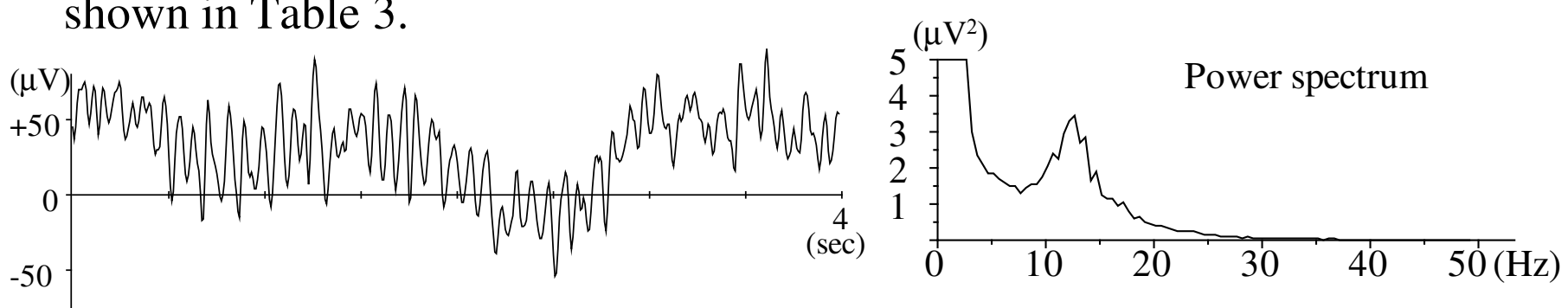

Control

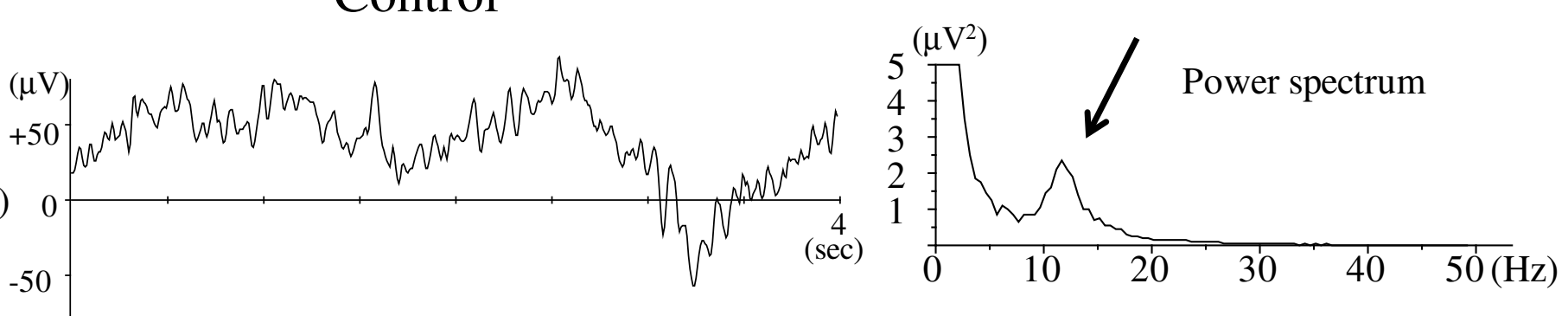

After remifentanil

[Fig 2] Effects of remifentanil on EEG during propofol anesthesia in 40yr old female

\begin{tabular}{|l|c|c|}
\hline & Amplitude $(\mu \mathrm{V})$ & $\alpha$ power $\left(\mu \mathrm{V}^{2}\right)$ \\
\hline Control & $13.1 \pm 2.6$ & $20.7 \pm 14.2$ \\
\hline After remifentanil & $10.7 \pm 2.3 *$ & $10.2 \pm 8.1^{*}$
\end{tabular}

[Table 3] Effects of remifentanil on EEG during propofol anesthesia $(\mathbf{N}=25) \quad * \mathbf{p}<0.001$

Discussion: Current result showed that remifentanil decreased $\alpha$ power, namely spindle activity, and amplitude of EEG during propofol anesthesia at non-surgical state.

As we don't examine whether fentanyl decreases spindle activity during propofol anesthesia, we can't clarify that the effect of remifentanil on spindle activity was remifentanil specific or common in opioid. However, we felt in our daily clinical practice that EEG amplitude during propofol-remifentanil anesthesia was smaller than that during propofol-fentanyl anesthesia. Then we think this phenomenon is remifentanil specific. At this point, the mechanism of this phenomenon is unclear.

We previously reported that noxious stimuli attenuated or diminished spindle activity, and it could be reversed by administration of fentanyl during isoflurane or sevoflurane anesthesia. We also observed the same phenomenon during propofol anesthesia in our daily clinical practice. And we actually use spindle activity as an indicator of adequate analgesia during surgery. In the point of view, this strategy is rather difficult during propofol-remifentanil anesthesia. Fortunately, we can easily get adequate analgesia by infusing remifentanil 0.2-0.3 $\mu \mathrm{g} / \mathrm{kg} / \mathrm{min}$. This phenomenon is not so serious in our clinical practice.

Conclusion: We found that EEG amplitude became smaller when remifentanil was co-administered with propofol at surgical level of anesthesia. Remifentanil seemed to attenuate the spindle activity at this anesthetic level. However, the mechanism is unclear.

\section{References}

1. Hagihira $S$, et al. Anesthesiology 2004;100:818-25

2. Kang H, et al. J Anesth 2017; DOI 10.1007/s00540-017-2319-1

3. Yufune S, et al. Br J Anaesth 2011;106:208-14

[Table 2] Demographic data 